\title{
Longitudinal space charge effect in slowly converging or diverging relativistic beams
}

\author{
Karl L. F. Bane and Alexander W. Chao \\ Stanford Linear Accelerator Center, Stanford University, Stanford, California 94309
}

(Received 20 July 2002; published 16 October 2002)

\begin{abstract}
Beginning with the Green function for a rod beam in a round beam pipe we derive the space charge induced average energy change and rms spread for relativistic beams that are slowly converging or diverging in round beam pipes, a result that tends to be much larger than the $1 / \gamma^{2}$ dependence for parallel beams. Our results allow for beams with longitudinal-transverse correlation, and for slow variations in beam pipe radius. We calculate, in addition, the space charge component of energy change and spread in a chicane compressor. This component indicates source regions of coherent synchrotron radiation (CSR) energy change in systems with compression. We find that this component, at the end of example compressors, approximates the total induced voltage obtained by more detailed CSR calculations. Our results depend on beam pipe radius (although only weakly) whereas CSR calculations do not normally include this parameter, suggesting that results of such calculations, for systems with beam pipes, are not complete.
\end{abstract}

DOI: 10.1103/PhysRevSTAB.5.104401

PACS numbers: 29.27.Bd, 29.27.Fh

\section{INTRODUCTION}

The Sub-Picosecond Photon Source (SPPS) is a new modification of the Stanford Linear Accelerator that promises to generate high peak current, femtosecond electron bunches that will then pass through an undulator to produce synchrotron light [1]. At the heart of the project is a magnetic chicane that compresses an $\mathrm{rf}$ gun generated bunch by more than a factor of 20 . To achieve the performance goals of the SPPS it is important that coherent synchrotron radiation (CSR) generated in the chicane not increase the beam energy spread and through it the emittance of the beam by a significant amount. This requirement places a tight tolerance $(\sim 5 \times$ $10^{-5}$ ) on the increase in energy spread allowed in the chicane.

CSR in compressors is recently a very active research topic (see, e.g., Ref. [2] and references contained therein). The CSR force has both longitudinal and transverse components, with the longitudinal component - the one that we will be interested in comparing with - thought to typically dominate in compressors [3]. One component of the longitudinal CSR force, which we here call the "space charge fields," generates an energy change in the beam equal to the negative of the potential difference between an initial and a final state of the beam; it is the component termed the "compression work" by Dohlus when applied to compressors [4]. (Note that another part of the fields, also called space charge, that depends on $1 / \gamma^{2}$, with $\gamma$ the Lorentz energy factor, and that is small for relativistic beams will not be considered here.) In this report we will focus on the space charge component, though, in general, one needs to consider all components to understand the longitudinal fields, for example, in a chicane. In the case of a converging beam in a drift tube, where the angles are small, this component will approximate the total longitudinal effect. In the case of a beam in a chicane this component is only a part, though — as we shall see - an important part, of the total effect.

Chicane compressors are beam lines with three or four bends separated by drifts. In the drift region between the last two bends the beam converges in the horizontal plane, with a large difference between the initial and final beam size values. At the same time the longitudinal beam distribution is unchanging, with the bunch length near its minimum, compressed value. We expect the potential change in this region to be a significant part of the total change within the chicane. Consequently we take as a first model of the chicane a relativistic converging beam in a beam pipe. Such a problem was studied by Zimmermann and Raubenheimer [5]. These authors noticed that in a converging (or diverging) relativistic beam the longitudinal space charge force no longer is proportional to $1 / \gamma^{2}$, and can therefore be a much larger effect than for the case of a parallel moving beam. They derive the space charge forces for bi-Gaussian beams beginning with the Bassetti-Erskine potentials and calculate the work done by these forces. Our approach here is different, beginning with the Green function for the potential of a rod beam oriented parallel to the axis in a circular beam tube, and we study several more aspects of the problem.

In this report we first study the longitudinal space charge effect of converging/diverging relativistic beams in a circular beam pipe and apply the results to the drift region before the last bend in the SPPS chicane. We then calculate the space charge part of the induced energy spread over the entire SPPS chicane beam line.

\section{A. Motivation}

As a well-known example where space charge dominates the solution of a problem, consider a cylindrically symmetric step from a beam pipe of radius $a$ to one of radius $b$, with a gentle tapered transition between the two. 
There are no bends or other sources of radiation upstream. Now consider a relativistic Gaussian bunch with length $\sigma_{z}$ that traverses unchanged past this step. Yokoya [6] has shown that if $a a^{\prime} / \sigma_{z} \ll 1$, where $a^{\prime}$ is the slope of the tapers, the longitudinal impedance for this problem is purely imaginary with no net energy radiated, and the problem looks very much like a statics problem-even when $\sigma_{z} / a \ll 1$-in that the local effect depends approximately on the local properties of the beam and the boundary. In such a problem we expect the energy change of beam particles as they pass the step to be given by minus the potential energy change between the current and initial positions. That is, the energy change is given by (discussed in more detail later) $-2 e N \lambda_{z} \ln (b / a)$, with $e N$ the bunch charge and $\lambda_{z}$ the longitudinal line density.

We demonstrate this through numerical calculation with the time domain, wakefield calculating module of MAFIA [7] in Fig. 1. It is difficult for us to numerically model shallow tapers so we choose a taper angle of $45^{\circ}$, $a=1 \mathrm{~cm}, b=1.5 \mathrm{~cm}$, and $\sigma_{z}=2 \mathrm{~cm}$ (nevertheless, the phenomenon for this model is the same). In the figure we show the transition shape (a) and the wake experienced by the center of the beam as a function of longitudinal position $s$ (b). The numerically obtained energy change of the particle is $-0.145 \mathrm{~V} / \mathrm{pC}$, which agrees well with the above space charge formula result, $-0.146 \mathrm{~V} / \mathrm{pC}$. For the entire bunch, the average and rms energy change of the beam are also well approximated by the space charge component.

However, even at the other, highly radiative extreme for such a problem $\left(a a^{\prime} / \sigma_{z} \gg 1\right)$ the space charge component is important. It has been shown by Heifets and Kheifets that in this case the space charge component of the fields exactly equals the radiation component [8].

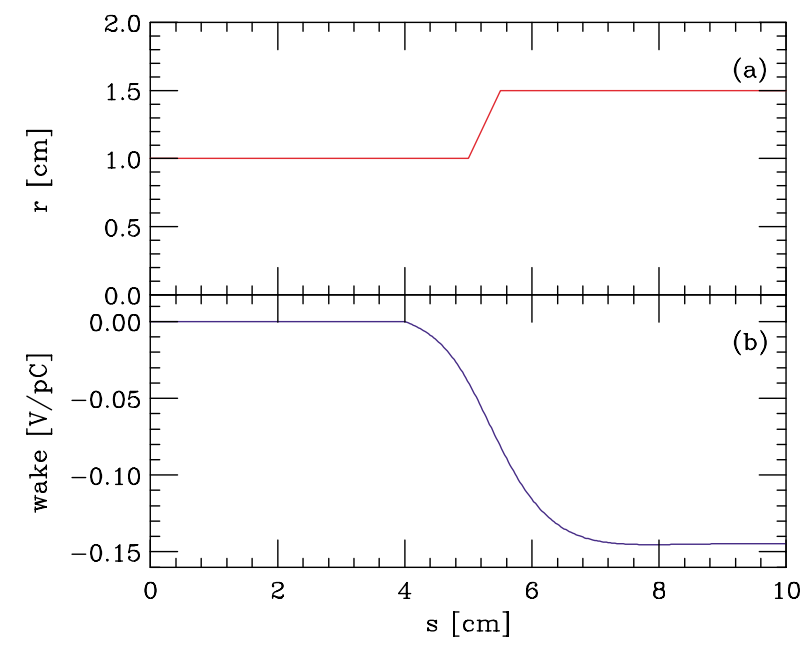

FIG. 1. (Color) Development of the wakefield at the center of a Gaussian bunch, with $\sigma_{z}=2 \mathrm{~cm}$, as it traverses on axis through a model transition. Shown are the transition geometry (a) and the numerically calculated wake (b).
However, unlike in the earlier case, here the beam will need to go far beyond the transition before the fields catch up to it and the total result is obtained.

Now consider a similar problem: a relativistic (transversely round) parallel beam, moving parallel to the axis in a round beam tube, is focused by a thin lens; after some distance it passes through a defocusing lens that transforms it back to a parallel beam, but this time, one with a smaller radius. One expects again that if the beam angle (after the first lens) is small, then the kinetic energy change from beginning to end is given by the negative of the change in potential energy; in addition, we expect the effect to be approximately static, in that the local effect depends on the local properties of the beam.

To obtain a first estimate of the size of the effect, consider now the simpler problem of two relativistic rod beams (no transverse extent) in free space, both with longitudinal distributions $\lambda_{z}$, that are comoving but at a slight angle $\theta$ toward each other. From Gauss's law we find that the gradient in energy change of a particle of one of the rods is given by $-2 e N \lambda_{z} \theta / d$, where $d$ is the distance to the other rod. Note that the size of the effect, instead of being on the order of $1 / \gamma^{2}$, is now on the order of $\theta$, which can be much larger. Using the Green function for the scalar potential of a rod moving parallel to the axis in a cylindrical pipe (presented in a following section) we obtain the same result for the two-rod problem. This example motivates us to solve the problem of relativistic converging/diverging beams, but now with transverse extent and including also the effect of the beam pipe walls.

\section{B. SPPS model problem}

As a concrete example we consider first the energy change in the beam as it traverses the drift space between the last two bends in the SPPS chicane. It is in this region of the chicane that the beam converges strongly in $x$ and that the bunch length is the smallest, and so we expect the space charge induced forces to be most significant here. Note that in this region the longitudinal distribution does not change. We ignore, for the moment, upstream effects. Later in the report we will consider also the energy spread change due to space charge throughout the compression process.

Between the last two bends of the chicane the beam tube length is $2.8 \mathrm{~m}$, and the length of the following (fourth) bend is $1.8 \mathrm{~m}$. For our model we will imagine the bend to be a thin lens and let the beam tube length $L=3.7 \mathrm{~m}$. The beam pipe radius is fixed at $a=7.62 \mathrm{~cm}$. We take the longitudinal charge distribution to be Gaussian, with a (constant) rms length of $\sigma_{z}=60 \mathrm{mi}-$ crons. In our model the vertical beam size remains fixed at $\sigma_{y}=100$ microns; the horizontal beam size begins at $\sigma_{x 0}=5.5 \mathrm{~mm}$ and ends at $\sigma_{x}=350 \mu \mathrm{m}$. In the real beam line there is an initial $x-z$ correlation in the 
TABLE I. Parameters for our SPPS model problem.

\begin{tabular}{lccc}
\hline \hline Bunch population & $N$ & 2.1 & $10^{10}$ \\
Bunch length & $\sigma_{z}$ & 60 & $\mu \mathrm{m}$ \\
Beam energy & $\mathcal{E}$ & 9 & $\mathrm{GeV}$ \\
Vertical beam size & $\sigma_{y}$ & 100 & $\mu \mathrm{m}$ \\
Horizontal beam size (initial) & $\sigma_{x 0}$ & 5.5 & $\mathrm{~mm}$ \\
Horizontal slice size (initial) & $\left(\sigma_{x 0}\right)_{s l}$ & 4.0 & $\mathrm{~mm}$ \\
Horizontal beam size (final) & $\sigma_{x}$ & 350 & $\mu \mathrm{m}$ \\
Beam tube length & $L$ & 3.7 & $\mathrm{~m}$ \\
Beam tube radius & $a$ & 7.62 & $\mathrm{~cm}$ \\
\hline \hline
\end{tabular}

distribution, which we will ignore at first. Other parameters are shown in Table I.

If the beam energy spread increases by $\sigma_{\delta}$ in the drift, then when it enters the bend the emittance will grow by (assuming the growth is small)

$$
\frac{\Delta \epsilon_{x}}{\epsilon_{x}} \approx \frac{\sigma_{\delta}^{2} \theta_{b}^{2} \beta_{x}}{2 \epsilon_{x}}
$$

with $\epsilon_{x}$ the horizontal emittance, $\theta_{b}$ the bend angle, and $\beta_{x}$ the beta function. If we take $\epsilon_{x}=2.8 \mathrm{~nm}, \theta_{b}=5.5^{\circ}$, $\beta_{x}=8 \mathrm{~m}$, then an energy spread increase of $\sigma_{\delta}=4 \times$ $10^{-5}$ results in an emittance growth $\Delta \epsilon_{x} / \epsilon_{x}=2 \%$, which we take as our tolerance. We will also use Eq. (1) to estimate emittance growth in a chicane, though, in this case, it is meant to be a rough guide only.

\section{ROUND BEAM}

Let us consider a relativistic bunch of positrons moving in a beam tube of radius $a$ from position $s_{0}$ to $s$. We are interested in beams that are converging or diverging in transverse dimension, but we assume that the angles are very small. The beam tube represents a continual electrical connection; its radius $a$ may vary slowly with longitudinal position $s$. We begin by assuming that the longitudinal distribution of the beam $\lambda_{z}(z)$ is arbitrary but frozen in time; however, we require that the bunch length $\sigma_{z}>a / \gamma$. The transverse distribution is assumed to be bi-Gaussian in horizontal and vertical positions $x$ and $y$, with respective rms beam sizes $\sigma_{x}, \sigma_{y}$, and centroids $\bar{x}, \bar{y}$. In principle, there can be correlation between $z$ and $(x, y)$ and the method is still valid. For simplicity we will assume no $x-y$ correlation.

Our basic assumptions are that radiation fields can be ignored, so that the energy change in the beam is given by a change in potentials, and that the angles are small, so that the local potential can be approximated by that of the translationally invariant problem. Let us begin with the case where the beam remains round and centered on the axis at all times, i.e., let $\sigma_{x}=\sigma_{y} \equiv \sigma$ and $\bar{x}=\bar{y}=0$ throughout. We assume that for any particle $r / \sigma$ remains fixed; i.e., any focusing field is linear and centered on the beam tube axis. For a test particle within the beam the electric field is dominantly in the radial direction and approximately given, by Gauss's Law, as (in cgs units)

$$
E_{r}(r) \approx \frac{2 e N \lambda_{z}}{r}\left(1-e^{-r^{2} / 2 \sigma^{2}}\right),
$$

with $e N$ the bunch charge and $r=\sqrt{x^{2}+y^{2}}$. The electric potential, with respect to the beam tube potential, is given by

$$
\phi(r)=\int_{r}^{a} E_{r}\left(r^{\prime}\right) d r^{\prime} \approx 2 e N \lambda_{z} \int_{r / \sigma}^{a / \sigma} \frac{\left(1-e^{-r^{\prime 2} / 2}\right) d r^{\prime}}{r^{\prime}} .
$$

The energy change of a test particle in moving from position $s_{0}$ to position $s$ is given by

$$
\Delta U=-\left.\phi(r, \sigma, a)\right|_{s_{0}} ^{s} \equiv-\Delta \phi .
$$

The energy change of a test particle can also be obtained from a path integral representing the work done by the field on the test particle:

$$
\Delta U=\int_{s_{0}}^{s} \vec{E} \cdot \vec{d} s
$$

(Since the force on the particle is obtained from a potential, any integration path between the end points will do.) For the round case, we can write

$$
\Delta U=\int_{s_{0}}^{s}\left(E_{r} \frac{d r}{d s}+E_{z}\right) d s,
$$

with $d r / d s$ a (small) constant and $E_{z}=-\partial \phi / \partial s$ the longitudinal electric field, given by (for $\sigma / a$ small)

$$
E_{z}=-2 e N \lambda_{z}\left(\frac{1}{a} \frac{d a}{d s}-\frac{e^{-r^{2} / 2 \sigma^{2}}}{\sigma} \frac{d \sigma}{d s}\right)
$$

(plus the familiar term that depends on $1 / \gamma^{2}$ ). Note that, even when $d a / d s=0$, two terms of the same order need to be considered in the work integral. Of the two methods of finding $\Delta U$, we feel that the earlier one is preferable, since it involves only knowledge of the potential at $s_{0}$ and $s$ and does not require an integration over $s$.

We see from the above equations that for the round case:

(i) For $\sigma / a$ small, at large $r(1 \ll r / \sigma<a / \sigma), \phi=$ $2 e N \lambda_{z} \ln (a / r)$.

(ii) If $r / \sigma=r_{0} / \sigma_{0}$ the energy change of the test particle is independent of $r$. If $e^{-a^{2} / 2 \sigma^{2}}$ is small compared to 1 , and $a$ is unchanged, then $\Delta U$ can be approximated as

$$
\Delta U=-2 e N \lambda_{z} \ln \left(\frac{\sigma_{0}}{\sigma}\right),
$$

where $\sigma_{0}$ is the initial beam size.

(iii) If $r / \sigma=r_{0} / \sigma_{0}$ and $a / \sigma=a_{0} / \sigma_{0}$, then there is no space charge energy change. For example, if the walls follow the change in beam size, there is no energy change. 
To find the total bunch energy change and the second moment of energy change we sum $\Delta \phi$ and $(\Delta \phi)^{2}$ over all particles. Note that, in general, for the average relative energy change $\langle\delta\rangle$ we do not need to know the correspondence between the positions of individual particles at $s_{0}$ and at $s$, but for the rms change $\delta_{\text {rms }}$ we do. (For a round beam, however, since $\Delta U$ is independent of $r$, this is not necessary.) For a round beam, with a Gaussian longitudinal distribution (assuming no $z-r$ correlation and $\sigma / a$ small) we obtain

$$
\langle\delta\rangle=-\frac{r_{e} N}{\sqrt{\pi} \bar{\gamma} \sigma_{z}} \ln \left(\frac{\sigma_{0}}{\sigma}\right),
$$

where $r_{e}=2.82 \times 10^{-15} \mathrm{~m}$, and $\bar{\gamma}$ is the average beam energy; $\delta_{\text {rms }}=0.39|\langle\delta\rangle|$.

\section{ELLIPTICAL BEAM}

The beam is usually not round but, rather, elliptical in cross section. The solution for elliptical beams is not so simple as for round beams. In general, we need to solve the Poisson equation numerically, with the boundary condition that at the beam pipe radius the potential is fixed. However, for the case of a round beam pipe we can easily find the Green function - the potential for a line charge - and then integrate over all the charges in the biGaussian transverse distribution.

The Green function at position $(x, y)$ within a round beam pipe of radius $a$, due to a line charge with charge distribution $e N \lambda_{z}$ parallel to the pipe axis and transverse offset $\left(x_{d}, y_{d}\right)$, can be shown to be

$$
\phi_{G}\left(x, y, x_{d}, y_{d}\right)=-e N \lambda_{z} \ln \left(\frac{a^{2}\left[\left(x-x_{d}\right)^{2}+\left(y-y_{d}\right)^{2}\right]}{\left(x_{d}^{2}+y_{d}^{2}\right)\left[\left(x-\frac{a^{2} x_{d}}{x_{d}^{2}+y_{d}^{2}}\right)^{2}+\left(y-\frac{a^{2} y_{d}}{x_{d}^{2}+y_{d}^{2}}\right)^{2}\right]}\right) .
$$

Written in this form, the connection with the method of images is clear: the image of a line charge with density $e N \lambda_{z}$ at $\left(x_{d}, y_{d}\right)$ is a line charge with density $-e N \lambda_{z}$ at $\left[\left(a^{2} x_{d}\right) /\left(x_{d}^{2}+y_{d}^{2}\right),\left(a^{2} y_{d}\right) /\left(x_{d}^{2}+y_{d}^{2}\right)\right]$; the extra factor of $\left(a^{2}\right) /\left(x_{d}^{2}+y_{d}^{2}\right)$ in the logarithm of Eq. (10) represents an additional constant potential contribution [9]. For a bi-Gaussian beam slice with rms extents $\sigma_{x}, \sigma_{y}$, and centroids $\bar{x}, \bar{y}$, the potential becomes

$$
\phi(x, y)=\frac{1}{2 \pi \sigma_{x} \sigma_{y}} \int_{-\infty}^{\infty} d x_{d} \int_{-\infty}^{\infty} d y_{d} \phi_{G}\left(x, y, x_{d}, y_{d}\right) e^{-\left(x_{d}-\bar{x}\right)^{2} / 2 \sigma_{x}^{2}-\left(y_{d}-\bar{y}\right)^{2} / 2 \sigma_{y}^{2}} .
$$

We assume here that the bi-Gaussian beam distribution is (almost) entirely contained within the beam pipe. When $\sigma_{x}=$ $\sigma_{y} \equiv \sigma$, Eq. (11) reduces to Eq. (3), our earlier result for a round beam.

Let us assume the beam size is small compared to $a$ and that the beam's distance from the wall is $\gtrsim \sigma_{x, y}$. Then from Eq. (11) we find the following.

(i) The potential of the slice, averaged over the transverse dimensions, is given by

$$
\langle\phi\rangle_{t} \approx e N \lambda_{z}\left[\mathbf{C}-2 \ln \left(\frac{\sigma_{x}+\sigma_{y}}{a}\right)+2 \ln \left(\frac{a^{2}-\bar{x}^{2}-\bar{y}^{2}}{a^{2}}\right)\right],
$$

with $\mathbf{C}=0.5772$, Euler's constant.

(ii) The potential at the center of the slice becomes

$$
\phi(\bar{x}, \bar{y}) \approx e N \lambda_{z}\left[\mathbf{C}+\ln 2-2 \ln \left(\frac{\sigma_{x}+\sigma_{y}}{a}\right)+2 \ln \left(\frac{a^{2}-\bar{x}^{2}-\bar{y}^{2}}{a^{2}}\right)\right] .
$$

Since the potential at the beam center does not equal the average potential, there must be a spread in potential; the rms in spread is on the order of $e N \lambda_{z} \ln 2$.

The potential itself can be approximated by

$$
\phi(x, y) \approx I(x, y)+\phi(\bar{x}, \bar{y})-2 e N \lambda_{z}\left[\frac{\bar{x}(x-\bar{x})+\bar{y}(y-\bar{y})}{a^{2}-\bar{x}^{2}-\bar{y}^{2}}\right],
$$

with

$$
I(x, y)=-e N \lambda_{z} \int_{0}^{\infty} \frac{1-\exp \left[-\frac{(x-\bar{x})^{2}}{2 \sigma_{x}^{2}+q}-\frac{(y-\bar{y})^{2}}{2 \sigma_{y}^{2}+q}\right]}{\sqrt{2 \sigma_{x}^{2}+q} \sqrt{2 \sigma_{y}^{2}+q}} d q .
$$

The advantage in using Eq. (14), instead of Eq. (11), for finding $\phi$ is that only one integral now needs to be solved; the disadvantage, however, is that we have lost generality in terms of beam size and location. Note that the last, centroid shift term in Eq. (14) typically is small. Note that if we set the first term in the integral Eq. (15) to zero, $I(x, y)$ becomes the familiar Bassetti-Erskine (BE) potential [10,11]. Note also that the electric field $\vec{E}(x, y)=-\nabla \phi(x, y)$ is given by the 
usual $\mathrm{BE}$ formula plus a new term due to the image charge, which is approximately given by

$$
2 e N \lambda_{z} \bar{r}^{2} \frac{\left(x \bar{r}^{2}-a^{2} \bar{x}\right) \hat{\mathbf{x}}+\left(y \bar{r}^{2}-a^{2} \bar{y}\right) \hat{\mathbf{y}}}{\left(\bar{r}^{2} x-a^{2} \bar{x}\right)^{2}+\left(y \bar{r}^{2}-a^{2} \bar{y}\right)^{2}}
$$

with $\bar{r}^{2}=\bar{x}^{2}+\bar{y}^{2}$.

If we transform the integral for $I$, it appears to become easier to solve numerically (for nonround beams). In
Eq. (15) let [10]

$$
t^{2}=\frac{2 \check{\boldsymbol{\sigma}}^{2}+q}{2 \hat{\boldsymbol{\sigma}}^{2}+q}
$$

where $\check{\sigma}(\hat{\sigma})$ is the minimum (maximum) of $\sigma_{x}$ and $\sigma_{y}$; let us also denote the corresponding coordinates $\check{q}(\hat{q})$ and average values $\check{\bar{q}}(\hat{\bar{q}})$. Equation (15) then becomes

$$
I(x, y)=-2 e N \lambda_{z} \int_{\check{\sigma} / \hat{\sigma}}^{1} \frac{1-\exp \left\{-\frac{\left(1-t^{2}\right)}{2\left(\hat{\sigma}^{2}-\check{\sigma}^{2}\right)}\left[(\hat{q}-\hat{\bar{q}})^{2}+(\check{q}-\check{\bar{q}})^{2} / t^{2}\right]\right\}}{1-t^{2}} d t .
$$

Unlike in the round beam case, $\Delta U$, for an elliptical beam moving from $s_{0}$ to $s$ in a linear system, is not independent of transverse position. The work performed on a test particle-assuming, as in our SPPS model example, only $x, \sigma_{x}$, and $\bar{x}$ change with $s$-becomes

$$
\Delta U=-\int_{s_{0}}^{s}\left(\frac{\partial \phi}{\partial x} \frac{d x}{d s}+\frac{\partial \phi}{\partial \sigma_{x}} \frac{d \sigma_{x}}{d s}+\frac{\partial \phi}{\partial \bar{x}} \frac{d \bar{x}}{d s}\right) d s .
$$

This is the approach used in Ref. [5] to obtain the space charge effect of converging beams.

\section{A. Averaged effect}

We are typically interested in the first and second moments of the energy change experienced by a beam in moving from $s_{0}$ to $s$. To obtain these moments we perform integrals of $\Delta \phi$ over the longitudinal and transverse (Gaussian) distributions. For the first moment we can simply subtract the average initial potential from the average final potential. For the second moment, however, we need to know the map for all particles between their initial and final states.
Let us here suppose, as in the SPPS model example, that the longitudinal distribution is fixed, and that the optics is linear, implying that for all particles $(x-\bar{x}) / \sigma_{x}$, $(y-\bar{y}) / \sigma_{y}$, remain fixed. Let us also suppose that there are no $x-y$ correlations, and, for the moment, that there also are no longitudinal-transverse correlations. From Eq. (12) we see that the average potential change over the beam is simply

$$
\langle\Delta \phi\rangle \approx-2 e N\left\langle\lambda_{z}\right\rangle\left[\ln \left(\frac{\sigma_{x}+\sigma_{y}}{\sigma_{x 0}+\sigma_{y 0}}\right)-\ln \left(\frac{a^{2}-\bar{x}^{2}-\bar{y}^{2}}{a^{2}-\bar{x}_{0}^{2}-\bar{y}_{0}^{2}}\right)\right],
$$

where subscript 0 indicates initial conditions. [From here on we will use brackets \langle\rangle to mean longitudinal average for functions of $z$, transverse average for functions of $(x, y)$, and average over both for functions of both.]

The second term in Eq. (20) gives the effect, due to the image charges, of the change in the beam offset. Note, however, that as long as the beam size changes, the first beam size term will tend to dominate over the second offset term. The second moment is given by

$$
\begin{aligned}
\left\langle\Delta \phi^{2}\right\rangle= & \frac{1}{(2 \pi)^{3 / 2} \sigma_{z}} \int_{-\infty}^{\infty} d z e^{-z^{2} / 2 \sigma_{z}^{2}} \int_{-\infty}^{\infty} d x_{r} \int_{-\infty}^{\infty} d y_{r} e^{-x_{r}^{2} / 2-y_{r}^{2} / 2} \\
& \times\left[\phi\left(x_{r} \sigma_{x}+\bar{x}, y_{r} \sigma_{y}+\bar{y}\right)-\phi_{0}\left(x_{r} \sigma_{x 0}+\bar{x}_{0}, y_{r} \sigma_{y 0}+\bar{y}_{0}\right)\right]^{2},
\end{aligned}
$$

where $\phi$ within the integral is given by Eq. (14). Since we assume no $x-z$ correlation the integral over $z$ can be done immediately, giving a result proportional to $e^{2} N^{2}\left\langle\lambda_{z}^{2}\right\rangle$. Note that in Eq. (21) we have made a change of variables from $x$ and $y$ to $x_{r}=(x-\bar{x}) / \sigma_{x}$ and $y_{r}=(y-\bar{y}) / \sigma_{y}$.

In general our calculations allow for the beam to both shift its centroid and change its shape transversely as it moves longitudinally from $s_{0}$ to $s$. Let us here consider the two effects separately. Consider the examples: (1) a beam that undergoes simple translation transversely, and (2) a beam that remains on axis but changes its shape transversely (as for the SPPS model example). For both examples the average potential change $\langle\Delta \phi\rangle$ is given by Eq. (20). As for the rms deviation, for example (1), the deviation within one slice of the beam is given by

$$
(\Delta \phi)_{s l, \mathrm{rms}}=\frac{2 e N \lambda_{z}}{a^{2}}\left[\left(\bar{x}-\bar{x}_{0}\right)^{2} \sigma_{x}^{2}+\left(\bar{y}-\bar{y}_{0}\right)^{2} \sigma_{y}^{2}\right]^{1 / 2} .
$$

This quantity is normally very small; it is second order in parameters offset over $a$ and beam size over $a$. Consequently, the rms deviation over the beam will tend to be dominated by the longitudinal variation in potential, giving

$$
(\Delta \phi)_{\mathrm{rms}} \approx \frac{\sqrt{\left\langle\lambda_{z}^{2}\right\rangle-\left\langle\lambda_{z}\right\rangle^{2}}}{\left\langle\lambda_{z}\right\rangle}|\langle\Delta \phi\rangle| .
$$

Note that if the longitudinal distribution is Gaussian, then 
$\left\langle\lambda_{z}\right\rangle=\left(2 \sqrt{\pi} \sigma_{z}\right)^{-1}$ and $\left\langle\lambda_{z}^{2}\right\rangle=\left(2 \sqrt{3} \pi \sigma_{z}^{2}\right)^{-1}$, which implies that $(\Delta \phi)_{\text {rms }} \approx 0.39|\langle\Delta \phi\rangle|$. Finally note that, since $\langle\Delta \phi\rangle$ itself depends on beam offset over $a$ to second order, as long as there is non-negligible beam size/orientation change between positions $s_{0}$ and $s$, the effect of beam offset can usually be ignored.

For example (2) the beam remains always on axis but changes its shape. For this situation, we see, from Eq. (14), that for any test particle in the beam the change in potential can be written in the form

$$
\Delta \phi=e N \lambda_{z}\left[f\left(x_{r}, y_{r}, \alpha_{0}, \alpha\right)-2 \ln \left(\frac{\sigma_{x}+\sigma_{y}}{\sigma_{x 0}+\sigma_{y 0}}\right)\right]
$$

where $x_{r}=(x-\bar{x}) / \sigma_{x}, y_{r}=(y-\bar{y}) / \sigma_{y}$, and $\alpha=\check{\sigma} / \hat{\sigma}$; $f\left(x_{r}, y_{r}, \alpha_{0}, \alpha\right)$ is a function only of the quantities indicated plus beam orientation (see below). Note that Eq. (24) implies that if both transverse beam sizes change by the same factor, then the variation in $\Delta \phi$ within one slice becomes zero. The average of $f$ over the Gaussians in $\left(x_{r}, y_{r}\right),\langle f\rangle=0$. It follows that

$$
(\Delta \phi)_{\mathrm{rms}}=e N\left[\left\langle f^{2}\right\rangle\left\langle\lambda_{z}^{2}\right\rangle+4\left[\left\langle\lambda_{z}^{2}\right\rangle-\left\langle\lambda_{z}\right\rangle^{2}\right] \ln ^{2}\left(\frac{\sigma_{x}+\sigma_{y}}{\sigma_{x 0}+\sigma_{y 0}}\right)\right]^{1 / 2}
$$

The first term in the large brackets gives the contribution of the transverse variation in $\Delta \phi$, the second term that of the longitudinal variation.

The results for $f_{\text {rms }}\left(=\sqrt{\left\langle f^{2}\right\rangle}\right)$ separate into two categories: (i) when the initial and final beam orientations are the same, and (ii) when they are different. This is evident since for the case when $\alpha$ remains unchanged, in case (i) $\left\langle f^{2}\right\rangle$ equals 0 (nothing has changed), whereas in case (ii) it does not. The result is

$$
f_{\text {rms }}= \begin{cases}\left|\ln \left[(1+\alpha) /\left(1+\alpha_{0}\right)\right]-\frac{1}{4} \ln \left[\left(1+\alpha^{2}\right) /\left(1+\alpha_{0}^{2}\right)\right]\right|: & \text { (case i), } \\ -\ln \left[(1+\alpha)\left(1+\alpha_{0}\right) / 4\right]+\frac{1}{4} \ln \left[\left(1+\alpha^{2}\right)\left(1+\alpha_{0}^{2}\right) / 4\right]: & \text { (case ii). }\end{cases}
$$

In Fig. 2 we plot $f_{\text {rms }}$ as a function of $\alpha_{0}$, for various values of $\alpha$. The solid lines give results for the case when the initial and final beam ellipses are oriented in the same way; the dashed lines give results for when they are not. Note that $f_{\text {rms }}$ is always $\leq 1.04$, with the limit reached for a pencil beam that changes its orientation $\left(\alpha_{0}=\alpha \sim 0\right)$. In the case the first term in the large brackets of Eq. (25) is small compared to the second term, we can again approximate $(\Delta \phi)_{\text {rms }}$ by Eq. (23).

\section{B. Longitudinal-transverse cor relation}

At the end of the third bend in the SPPS chicane (the initial condition for our model problem) there is significant $x-z$ correlation in the beam. In the equivalent position in the second chicane of the LCLS project the beam is extremely correlated, and this fact must be taken into account for obtaining the space charge effect.

To include $x$ - $z$ correlations in our calculations we again assume that the potential of every slice (at fixed $z$ ) is independent of other slices. However, in calculations the slice beam size $\left(\sigma_{x}\right)_{s l}=\sqrt{\sigma_{x}^{2} \sigma_{z}^{2}-\sigma_{x z}^{2}} / \sigma_{z}$ now takes over the function that the total beam size $\sigma_{x}$ has in the case of no correlations, and, in addition, the slices are offset in $x$ by an amount $\bar{x}=z \sigma_{x z} / \sigma_{z}^{2}$ (see Fig. 3). If, as usual, the beam size is small compared to $a$, and the beam is not near the wall, we expect, from the results of the previous section, that we can ignore the centroid shifts without affecting the average and rms energy change significantly. We demonstrate in this section that this is true for the SPPS model parameters.

In general, to calculate $\left\langle\Delta \phi^{2}\right\rangle$ for a beam with $x-z$ correlation we numerically solve Eq. (21), but substituting the slice offsets $\bar{x}(z), \bar{x}_{0}(z)$, in the arguments for $\phi, \phi_{0}$, in the integrand. In the most general calculation we use $\phi$ as given in Eq. (11). Then we have five integrals to do. However, since $\phi, \phi_{0}$, are smooth functions, well approximated by low order polynomials, we can perform the three outer integrals (over $z, x_{r}, y_{r}$ ) efficiently using Hermite numerical quadrature.

Let us now consider the SPPS model example: we have initially $\sigma_{x 0}=5.6 \mathrm{~mm}, \sigma_{y 0}=100 \mu \mathrm{m}$, and finally $\sigma_{x}=$ $350 \mu \mathrm{m}, \sigma_{y}=100 \mu \mathrm{m}$; other beam parameters can be found in Table I. Initially there is $x-z$ correlation with $\left(\sigma_{x 0}\right)_{s l}=0.73 \sigma_{x 0}$, but in the final state there is no correlation. To show the sensitivity to initial $x$ - $z$ correlation we

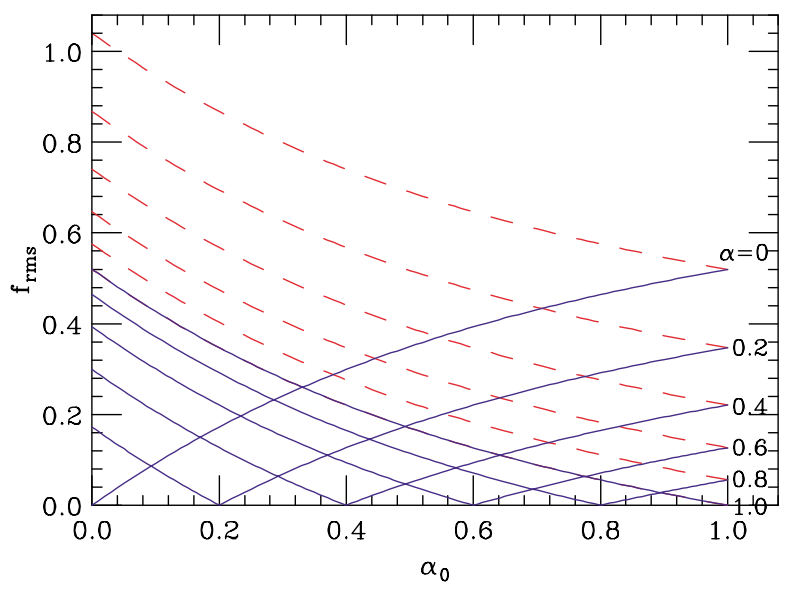

FIG. 2. (Color) The rms of the auxiliary function $f$, as a function of $\alpha_{0}$, for various values of $\alpha$. The solid lines give results for the case when initial and final ellipses are oriented in the same way; the dashed lines give results for when they are not. 


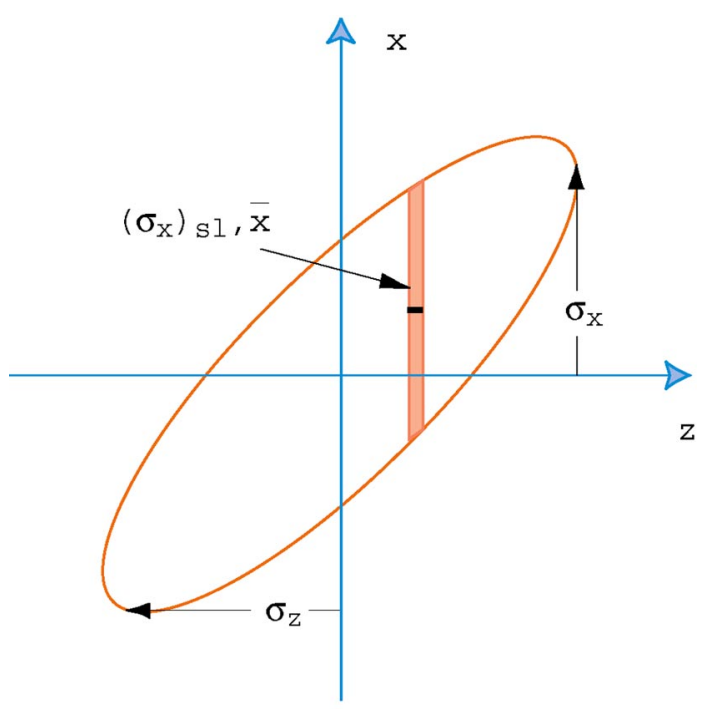

FIG. 3. (Color) Sketch of beam with $x-z$ correlation, showing the slice parameters $\left(\sigma_{x}\right)_{s l}, \bar{x}$, and the beam parameters $\sigma_{x}, \sigma_{z}$.

plot, in Fig. 4, the relative rms energy change $\delta_{\text {rms }}$ as a function of $\left(\sigma_{x 0}\right)_{s l}$ (keeping $\sigma_{x 0}$ fixed) as obtained by the general numerical calculation. At the upper end of the curve $\left(\sigma_{x 0}\right)_{s l}=\sigma_{x 0}$, there is no correlation; at the lower, maximum correlation end, $\left(\sigma_{x 0}\right)_{s l}$ equals the final beam size $\left(\sigma_{x}\right)$, and the slice beam size does not change. Also shown in the figure, by the dashes, is $-0.39\langle\delta\rangle$.

The interaction decreases as $\left(\sigma_{x 0}\right)_{s l}$ decreases, because the change in (slice) beam size from initial to final state is reduced; the centroid offset of the slices has little effect. We see this, for example, at $\left(\sigma_{x 0}\right)_{s l}=\sigma_{x}\left(=0.064 \sigma_{x 0}\right.$, no beam size change and maximum correlation) $\delta_{\text {rms }} \sim 6 \times$ $10^{-8}$, which is very small. We repeated the calculation, but now setting the slice offsets to zero and using the

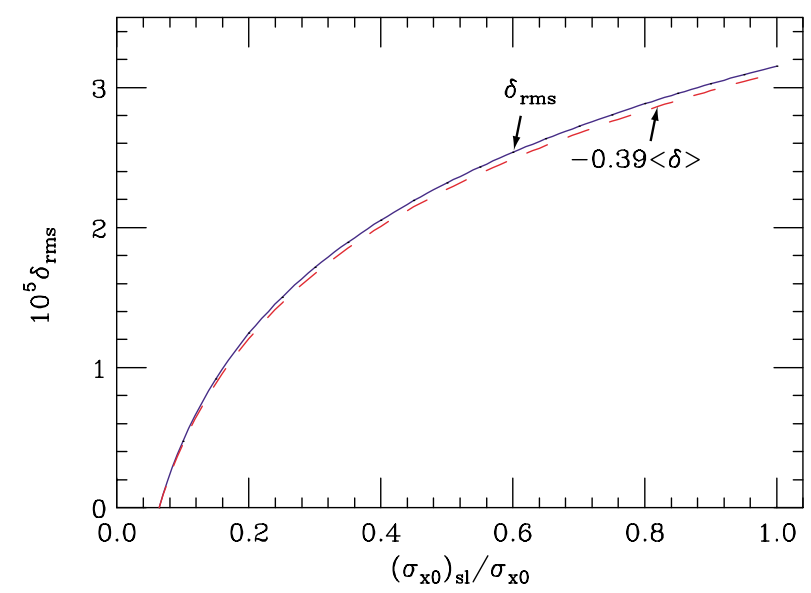

FIG. 4. (Color) For the SPPS model parameters: sensitivity to correlation component in initial beam size. Given are $\delta_{\text {rms }}$ (solid line) and $-0.39\langle\delta\rangle$ (dashed line) as functions of $\left(\sigma_{x 0}\right)_{s l} / \sigma_{x 0}$ keeping $\sigma_{x 0}$ fixed.

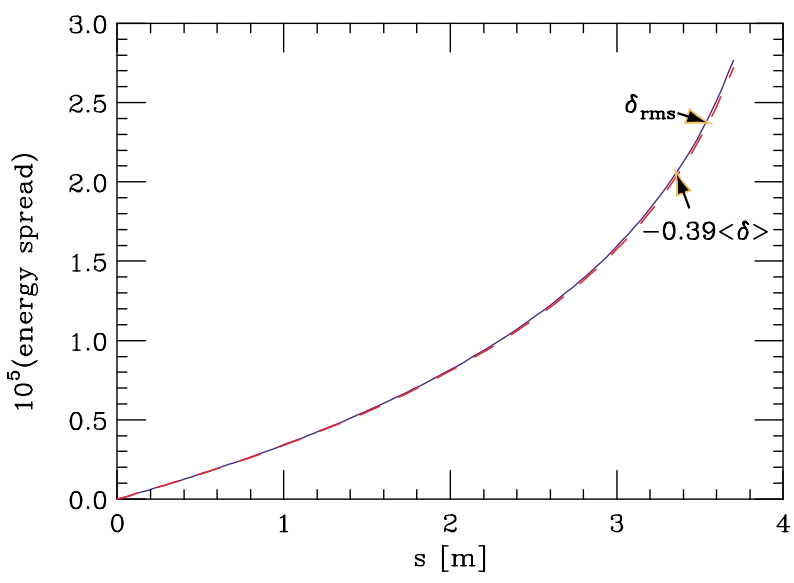

FIG. 5. (Color) The development of the rms energy spread for the SPPS model problem (solid line) and the quantity $-0.39\langle\delta\rangle$ (dashed line).

simpler Eqs. (20), (25), and (26). We obtained essentially the same curves as before. For the SPPS model, $\left(\sigma_{x 0}\right)_{s l}=$ $0.73 \sigma_{x 0}$, and thus $\langle\delta\rangle=-7.1 \times 10^{-5}$ and $\delta_{\text {rms }}=2.8 \times$ $10^{-5}$, which is within, but close to, our tolerance, $4 \times$ $10^{-5}$. Note that the transverse component of rms relative energy is only $3.5 \times 10^{-6}$.

In Fig. 5 we plot the development of $\delta_{\text {rms }}$ for our SPPS example problem (the solid curve) and $-0.39\langle\delta\rangle$ (the dashed curve). The two curves are very close, indicating that the (weighted) transverse variation in energy change in the bunch is always small compared to the longitudinal variation. Finally, we plot in Fig. 6 a contour plot showing the change in $\delta$, between the beginning and end of the SPPS drift, over the central slice of the beam. Shown is the energy change over a $\pm 5 \sigma$ rectangle in the $x, y$ plane.

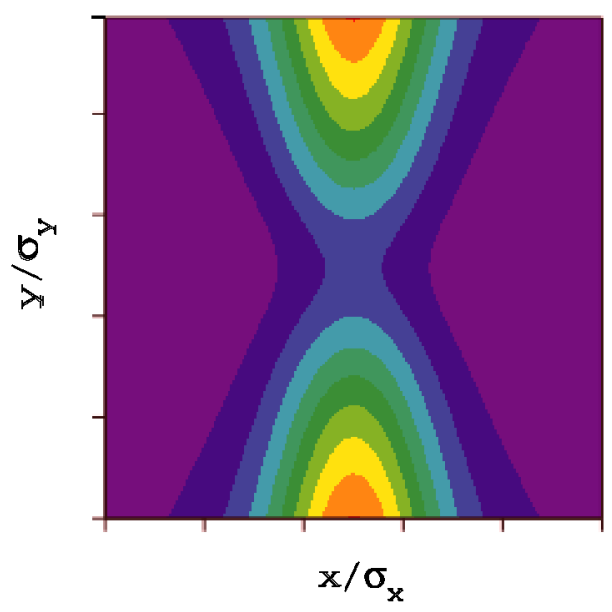

FIG. 6. (Color) Contour plot of energy change, over the central slice of the beam, between the beginning and end of the SPPS model problem. Results are shown over a $\pm 5 \sigma$ rectangle in $x$ and $y$. The peaks (orange) are at $\delta=-6.5 \times 10^{-5}$, the floor (violet) is at $\delta=-11 . \times 10^{-5}$. 
There is a difference of $\delta=4.5 \times 10^{-5}$ between the maximum and minimum values in the plot (remember that for the round beam case $\Delta \phi$ was independent of transverse position).

\section{Reducing the effect}

Suppose we would like to reduce the induced energy spread. How could we do it? For the round beam case we saw that in a linear optics region (in such a way that $r / \sigma$ is constant), if $a$ is allowed to vary adiabatically such that $\sigma / a$ is the same at $s_{0}$ as at $s$, then the induced energy spread is zero. The same can be shown to be true for the elliptical beam in a cylindrical beam tube, as long as $a$, $\sigma_{x}, \sigma_{y}, \bar{x}$, and $\bar{y}$ (though the last two are not usually important) all change under identical scaling. For the elliptical beam case, we see from Eq. (12) that having a different beam tube radius at $s$ than at $s_{0}\left(=a_{0}\right)$, the longitudinal space charge effect at the test particle is modified (neglecting the usually small, centroid offset term) by the quantity

$$
\Delta U=-2 e N \lambda_{z} \ln \left(\frac{a}{a_{0}}\right)
$$

(This equation is also valid for the round beam case.) For the elliptical beam, by only adjusting $a$, we can again reduce the space charge effect, though this time we cannot reduce it to zero. We find that for the SPPS model problem the induced energy spread is reduced by a factor of 2 if at the end $a$ is reduced (from $7.62 \mathrm{~cm}$ ) to $2.5 \mathrm{~cm}$; it is reduced to its minimum, $\delta_{\mathrm{rms}}=4 . \times 10^{-6}$ if at the end $a=0.8 \mathrm{~cm}$.

\section{BUNCH COMPRESSION}

Up to now we have limited ourselves to the case of converging/diverging beams in drift regions, where the longitudinal bunch distribution remains fixed. Let us extend our space charge calculations to allow compression. There will be a radiation contribution to the beam's energy change while in a compressor, though our calculation will not include it. We still assume that the beam pipe is round, with the same radius $a$, and that the pipe represents a continuous electrical connection.

The change in average potential is again simple to calculate. We see from Eq. (12) that (if $a$ does not change) it is given by

$$
\langle\Delta \phi\rangle=e N\left\{\left\langle\lambda_{z}\right\rangle\left[\mathbf{C}-2 \ln \left(\frac{\sigma_{x}+\sigma_{y}}{a}\right)\right]-\left\langle\lambda_{z 0}\right\rangle\left[\mathbf{C}-2 \ln \left(\frac{\sigma_{x 0}+\sigma_{y 0}}{a}\right)\right]\right\} .
$$

Note that a change in bunch length has a bigger effect than a change in transverse beam size.

The second moment $\left\langle\Delta \phi^{2}\right\rangle$ is more difficult to obtain than before because, for any test particle, $\lambda_{z}$ at the initial and final positions is different, and because the phase advance between the two positions is not normally an integer multiple of $2 \pi$, so-in addition to $x, y, z$ - one needs to also average over $x^{\prime}, y^{\prime}, \delta$. The most general solution is given by

$$
\left\langle\Delta \phi^{2}\right\rangle=\int \ldots \int \mathbf{d} \mathbf{x}_{0} d x_{d 0} d y_{d 0} d z_{d 0} \psi_{0}\left(\mathbf{x}_{0}\right)\left[\tilde{\phi}_{G}\left(x, y, z, x_{d}, y_{d}, z_{d}\right)-\tilde{\phi}_{G}\left(x_{0}, y_{0}, z_{0}, x_{d 0}, y_{d 0}, z_{d 0}\right)\right]^{2},
$$

where $\mathbf{x}_{0}$ represents a six-dimensional vector $\left(x_{0}, x_{0}^{\prime}, y_{0}, y_{0}^{\prime}, z_{0}, \delta_{0}\right), \psi_{0}$ is the initial six dimension beam distribution, and the Green function $\tilde{\phi}_{G}$ is related to the one introduced in Eq. (10) by $\tilde{\phi}_{G}=\delta\left(z-z_{d}\right) \phi_{G}$. Note that to solve this equation we need to perform eight integrals (plus one integral over a delta function). To solve the integrals of Eq. (29) we need to substitute $\mathbf{x}=M \mathbf{x}_{0}$, where the symplectic, first order transfer matrix $M$ is of the form [12] (we assume our optics is linear)

$$
M=\left[\begin{array}{cccccc}
R_{11} & R_{12} & 0 & 0 & 0 & R_{16} \\
R_{21} & R_{22} & 0 & 0 & 0 & R_{26} \\
0 & 0 & R_{33} & R_{34} & 0 & 0 \\
0 & 0 & R_{43} & R_{44} & 0 & 0 \\
R_{51} & R_{52} & 0 & 0 & 1 & R_{56} \\
0 & 0 & 0 & 0 & 0 & 1
\end{array}\right]
$$

This matrix assumes bending in $x$ only. Note that if initially the beam is in a dispersion-free region (such as at the beginning of the SPPS chicane), then $R_{16}=\eta, R_{26}=\eta^{\prime}, R_{51}=R_{21} \eta-R_{11} \eta^{\prime}, R_{52}=-R_{12} \eta^{\prime}+R_{22} \eta$, and $R_{56}=$ $-\int_{s_{0}}^{s} d s \eta / \bar{\rho}$, with $\eta$ the dispersion function and $\bar{\rho}$ the bending radius. For the SPPS chicane we take the initial beam distribution to be

$$
\psi_{0}\left(\mathbf{x}_{0}\right)=\rho_{x x^{\prime} 0}\left(x_{0}, x_{0}^{\prime}\right) \rho_{y y^{\prime} 0}\left(y_{0}, y_{0}^{\prime}\right) \lambda_{z 0}\left(z_{0}\right) \lambda_{\delta 0}\left(\delta_{\text {in }}+\delta_{\text {corr }}^{\prime} z_{0}\right),
$$

where $\rho$ 's and $\lambda$ 's, represent, respectively, correlated bi-Gaussian and Gaussian distributions; where $\delta_{\text {in }}$ and $\delta_{\text {corr }}^{\prime} z_{0}$ represent the (with $z$ ) uncorrelated and correlated components of initial energy spread.

From our earlier results we know that we can simplify the calculation of $\left\langle\Delta \phi^{2}\right\rangle$ by using the potential given in Eq. (14) (let us denote it by $\phi_{\mathrm{BE}}$ ), where, in addition, we can usually drop the third, beam-offset term. We are left with 
the six dimensional integral

$$
\left\langle\Delta \phi^{2}\right\rangle=\int \ldots \int \mathbf{d} \mathbf{x}_{0} \psi_{0}\left(\mathbf{x}_{0}\right)\left\{\phi_{\mathrm{BE}}\left[x-\bar{x}, y, z,\left(\sigma_{x}\right)_{s l}, \sigma_{y}, \sigma_{z}\right]-\phi_{\mathrm{BE}}\left[x_{0}-\bar{x}_{0}, y_{0}, z_{0},\left(\sigma_{x 0}\right)_{s}, \sigma_{y 0}, \sigma_{z 0}\right]\right\}^{2}
$$

With this method, however, to perform the integrals we need $\sigma_{y}, \sigma_{z},\left(\sigma_{x}\right)_{s l}$, and $\bar{x}$ (the final slice rms size and offset), as functions of the initial beam distribution. They are obtained by $\boldsymbol{\sigma}=M \boldsymbol{\sigma}_{0} M^{T}$, with $M^{T}$ the transpose of $M$, which converts the initial covariance matrix of the beam to the final covariance matrix, and then by $\left(\sigma_{x}\right)_{s l}=\sqrt{\sigma_{x}^{2} \sigma_{z}^{2}-\sigma_{x z}^{2}} / \sigma_{z}$ and $\bar{x}=z \sigma_{x z} / \sigma_{z}^{2}$.

For an efficient calculation of Eq. (32) we first calculate the beam covariance matrix at position $s$. Knowing $\left(\sigma_{x}\right)_{s l}$ and $\sigma_{y}$ we calculate the transverse dependence of $\phi_{\mathrm{BE}}$ over a $\pm 7 \sigma$ two-dimensional grid. Then, when needed by the integrals, we use results splined to this grid. As for the integrals over phase space, if we assume Gaussian distributions in all directions, these can again be performed efficiently using Hermite quadrature [13]. If we take seven terms for each integral, then we need calculate only $10^{5}$ terms in all.

\section{The SPPS chicane}

For the SPPS chicane we take initial bunch length $\sigma_{z 0}=1.15 \mathrm{~mm}$, initial uncorrelated energy spread $\sigma_{\delta \text { in }}=5.5 \times 10^{-4}$, correlated energy parameter $\delta_{\text {corr }}^{\prime}=$ $0.0133 \mathrm{~mm}^{-1}$, and final $R_{56}=7.5 \mathrm{~cm}$, which result in final bunch length $\sigma_{z f}=41 \mu \mathrm{m}$. Our results for the space charge energy change, from the beginning to any position within the SPPS chicane, are shown in Fig. 7. Shown are $\delta_{\text {rms }}$ and $\langle\delta\rangle$ (top frame), and also the development of the beam sizes (bottom frame). Note that $\sigma_{y}$ (not shown) varies nearly linearly from an initial $55 \mu \mathrm{m}$ to a final $110 \mu \mathrm{m}$. The bend regions are marked by orange bands. Note that within the bends of the SPPS chicane the beam pipe is not round, and our space charge results are not valid. Nevertheless, in the drift regions and up to the edge of the bends they are valid. We see that everywhere $-0.39\langle\delta\rangle$ is a good approximation of $\delta_{\text {rms }}$, again suggesting that the longitudinal variation in $\delta$ dominates over the transverse variation. In addition, we see that most of the effect occurs within bends 3 and 4 . At the end our results are $\delta_{\text {rms }}=11.5 \times 10^{-5}$ and $\langle\delta\rangle=-27 \times 10^{-5}$. We see that our earlier model problem results do not give a good approximation for space charge induced spread in the fourth bend, since the energy spread increases significantly already in the third bend. Our rough estimate for emittance growth, Eq. (1), using the rms energy spread at the center of the last bend $\left(10 \times 10^{-5}\right)$, yields $\sim 13 \%$, which is much larger than our tolerance.
Let us compare our results with the energy spread obtained from CSR calculations that include also radiation terms. These CSR calculations, however, assume the beam to be in free space. If we, equivalently, let $a=\gamma \sigma_{z}$ our results become $\delta_{\text {rms }}=16 \times 10^{-5}$ and $\langle\delta\rangle=-37 \times 10^{-5}$. The CSR calculation results are $\delta_{\text {rms }}=16 \times 10^{-5}$ and $\langle\delta\rangle=-23 \times 10^{-5}$ (though these parameters are still increasing in amplitude at the end of the calculation, $5 \mathrm{~m}$ beyond the fourth bend) [14]. This suggests that, in a chicane compressor, the space charge component of energy spread can dominate the total. A significant difference, however, is that for the space charge component most of the growth happens within the last two bends, while for the total, the energy spread accumulates gradually, with a significant contribution occurring in the drift regions downstream of these bends. We expect the total CSR energy loss after all the fields have caught up to the beam to be equal or greater than the space charge component ("the compression work") [4].

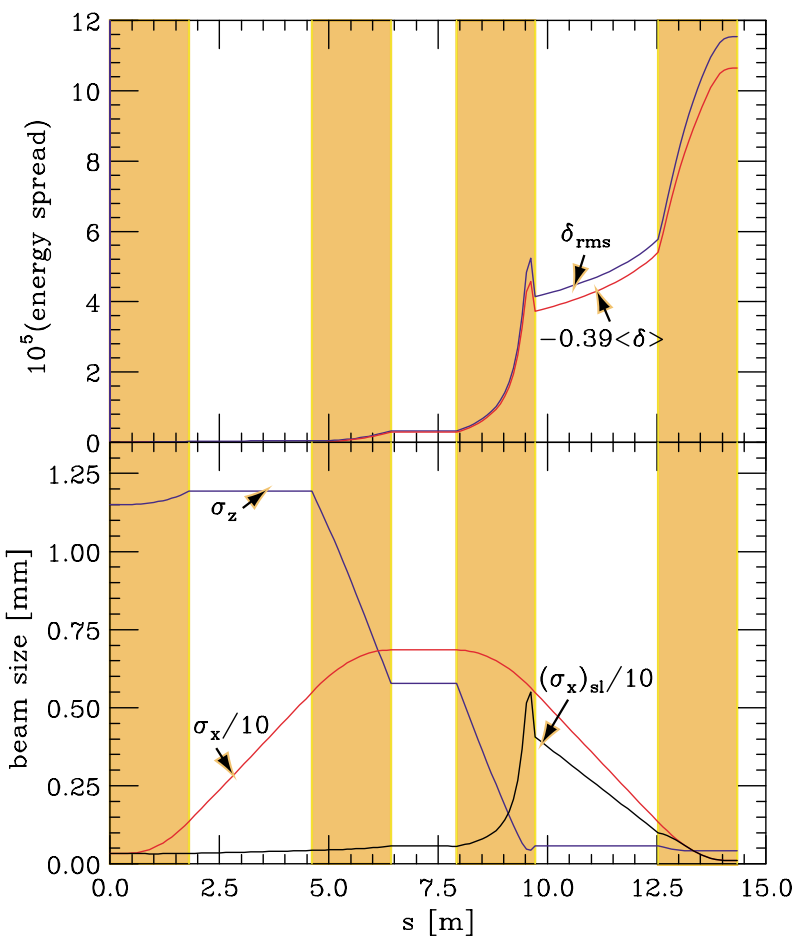

FIG. 7. (Color) Space charge component of energy spread in the SPPS chicane. Shown are $\delta_{\text {rms }}$ and $\langle\delta\rangle$ (top frame), and also the development of the beam sizes (bottom frame). The bend regions are marked in orange. Note that within the bends, our space charge results, which assume a round beam pipe, are not valid. 
Note that, due to the catch-up requirement, an estimate for emittance growth (within the last bend) using only the space charge component of energy spread will tend to be pessimistic.

Finally, in March 2002 a meeting was held in Berlin, CSR-Workshop-2001, to discuss coherent synchrotron radiation [15]. For the purpose of comparing simulations, benchmark chicane parameters were generated. In the Appendix we give the space charge component of energy spread for one of these examples. Our results are comparable for the total CSR induced energy change, as presented at the workshop (unfortunately $\delta_{\text {rms }}$, as presented at the workshop, is not given in a form that is easy for us to compare).

\section{CONCLUSION}

Beginning with the Green function for a rod beam oriented parallel to the axis of a round beam pipe we have derived the space charge induced energy shift and spread for relativistic beams that are slowly converging or diverging, a result that tends to be larger than the $1 / \gamma^{2}$ dependence for parallel beams. In a simple drift region our results give the change in (kinetic) energy spread in the beam. We have found that the variation in energy is typically dominated by the longitudinal variation, so that for Gaussian beams the rms energy spread $\delta_{\text {rms }} \approx$ $|0.39\langle\delta\rangle|$, with $\langle\delta\rangle$ the average energy change. We have found that in calculations for converging/diverging beams with an $x-z$ correlation the result is essentially the same as for an on-axis beam with no correlation, but with the horizontal beam size $\sigma_{x}$ replaced by the slice beam size $\left(\sigma_{x}\right)_{s l}$. We have found that by varying the beam pipe radius to roughly follow the beam size variation the space charge effect can be reduced.

We have extended the calculation to systems with bends, such as chicane compressors, but now our results give only part of the total energy spread induced in the beam. Nevertheless, our results can be used to indicate source regions of CSR induced energy spread. Comparing with more complete CSR calculation results, it appears that due to bunch compression the space charge component can dominate the total longitudinal effect, after enough time is given for the radiation fields to catch up with the beam. Because of the catch-up requirement, space charge estimates of energy spread and emittance growth in the last bend of a chicane will tend to be too large. We note, in addition: (i) even in a chicane system $\delta_{\text {rms }} \approx|0.39\langle\delta\rangle|$ (for Gaussian beams), which allows us to obtain an estimate of $\delta_{\text {rms }}$ using a simple formula; (ii) our results are not valid within nonround beam pipe regions, such as is usually the case in bends; (iii) our results depend on beam pipe radius $a$ (although only weakly), whereas CSR calculations do not normally include this parameter, suggesting that results of such calculations, for systems with beam pipes, are not complete.

From our space charge calculations for the SPPS chicane we obtain a final induced energy spread of $\delta_{\text {rms }}=$ $1.1 \times 10^{-4}$, though this result should be taken as an overestimate.

\section{ACKNOWLEDGMENTS}

The authors thank S. Heifets, V. Ivanov, and G. Stupakov for helpful discussions on the subject of space charge forces, and P. Emma for motivating this work, describing the SPPS project, and discussing the results. We also thank T. Raubenheimer for discussing his earlier work on this subject. This work was supported by the Department of Energy, Contract No. DE-AC0376 SF00515.

\section{APPENDIX: BERLIN BENCHMARK CHICANE}

In March 2002 there was a meeting in Berlin to discuss coherent synchrotron radiation [15]. For the purpose of comparing simulations, parameters for benchmark chicanes were generated. Parameters for one such example are given in Table II. We compute the space charge component of energy spread for this example. No beam pipe radius is specified, and we take, for example, $a=$ $7.62 \mathrm{~cm}$. The results are shown in Fig. 8. Given are $\delta_{\text {rms }}$ and $\langle\delta\rangle$ (top frame), and $\sigma_{z}, \sigma_{x}$, and $\left(\sigma_{x}\right)_{s l}$ (bottom frame). We note that most of the space charge effect occurs in the third bend. We see that again $\delta_{\text {rms }}=$ $-0.39\langle\delta\rangle$ is a good approximation. This time, throughout most of the chicane, $\left(\sigma_{x}\right)_{s l}$ is very small compared to $\sigma_{x}$, implying that there is a large $x-z$ correlation.

The total space charge induced energy change $\langle\delta\rangle$ equals $-3.5 \times 10^{-4}$, which is comparable to the total energy change obtained by complete CSR calculations, $-(4.5-6.0) \times 10^{-4}$ (though the amplitude appears to still be increasing at the end of the CSR simulation) [16]. (Unfortunately the induced spread $\delta_{\text {rms }}$ in Ref. [16] is not given in a form that is easy for us to compare.) These CSR calculations, however, assume the beam to be in free space. If we, equivalently, let $a=\gamma \sigma_{z}$ our final results rise by about $10 \%$.

TABLE II. Parameters for a Berlin benchmark chicane.

\begin{tabular}{lccc}
\hline \hline Bunch population & $N$ & 6.25 & $10^{9}$ \\
Initial bunch length & $\sigma_{z 0}$ & 200 & $\mu \mathrm{m}$ \\
Final bunch length & $\sigma_{z}$ & 20 & $\mu \mathrm{m}$ \\
Beam energy & $\mathcal{E}$ & 5 & $\mathrm{GeV}$ \\
Initial energy correlation & $\delta_{\text {corr }}^{\prime}$ & 36 & $\mathrm{~m}^{-1}$ \\
Initial uncorrelated energy spread & $\sigma_{\delta \text { in }}$ & 2 & $10^{-6}$ \\
Compression parameter & $R_{56}$ & -2.5 & $\mathrm{~cm}$ \\
Emittance & $\epsilon_{x, y}$ & 0.1 & $\mathrm{~nm}$ \\
\hline \hline
\end{tabular}




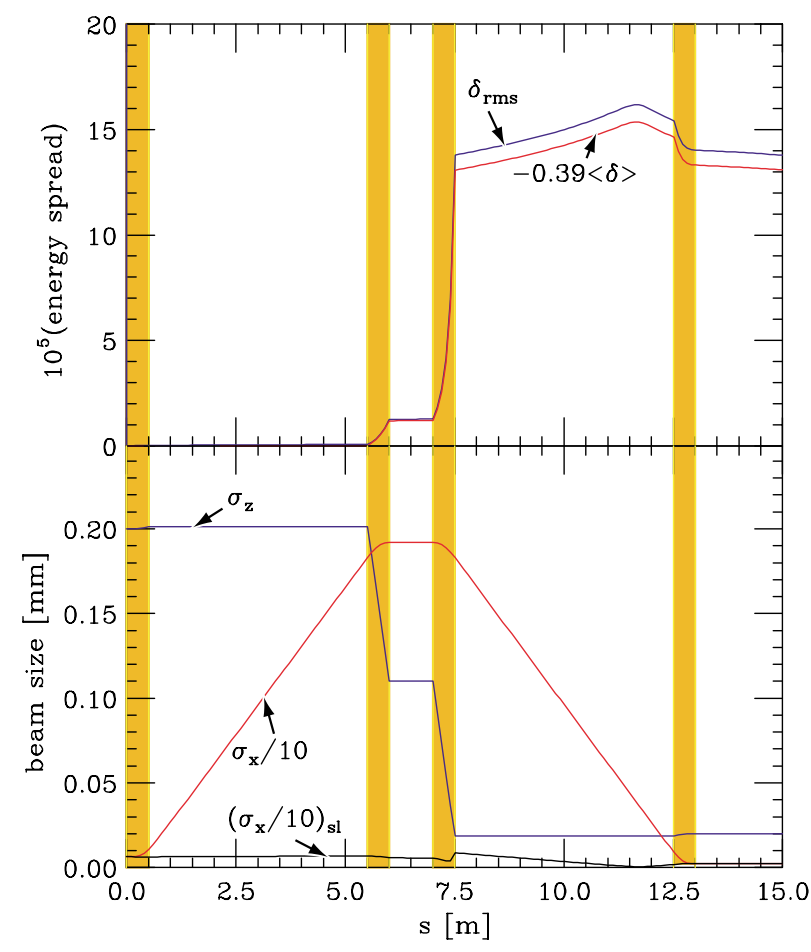

FIG. 8. (Color) Space charge component of energy spread in the Berlin benchmark chicane. Shown are $\delta_{\text {rms }}$ and $\langle\delta\rangle$ (top frame), and also the development of the beam sizes (bottom frame). The bend regions are marked in orange. Note that within the bends, our space charge results, which assume a round beam pipe, are not valid.

[1] P. Emma, R. Iverson, P. Krejcik, P. Raimondi, and J. Safranek, in Proceedings of the 2001 Particle
Accelerator Conference, Chicago, IL (IEEE, Piscataway, NJ, 2001), p. 4038.

[2] R. Li, in Proceedings of the 1999 Particle Accelerator Conference, New York, NY (IEEE, Piscataway, NJ, 1999), p. 118.

[3] R. Li, in The Physics of High Brightness Beams: Proceedings, Los Angeles, 1999 (World Scientific, Singapore, 2000).

[4] M. Dohlus, see copies of transparencies presented at the CSR-Workshop-2001 at http://www.desy.de/csr/ csr_workshop_2002.

[5] F. Zimmermann and T. Raubenheimer, Nucl. Instrum. Methods Phys. Res., Sect. A 390, 279 (1997).

[6] K. Yokoya, CERN Report No. CERN-SL 90-88 AP, 1990.

[7] The MAFIA Collaboration, User guide, CST-GmbH, Darmstadt, Germany.

[8] S. Heifets and S. Kheifets, Rev. Mod. Phys. 63, 631 (1991).

[9] J. D. Jackson, Classical Electrodynamics (John Wiley \& Sons, New York, NY, 1998), 3rd ed., p. 82, problem 2.11.

[10] M. Bassetti and G. A. Erskine, CERN Report No. ISRTH 80-06, 1980.

[11] S. Kheifets, DESY PETRA Note No. 119, 1976.

[12] A. Chao, see http://www.slac.stanford.edu/ achao/ LectureNotes.html.

[13] M. Abramowitz and I. Segun, Handbook of Mathematical Functions (Dover Publications, New York, NY, 1965), p. 890.

[14] L. Bentson, P. Emma, and P. Krejcik, in Proceedings of the 8th European Particle Accelerator Conference, Paris, France, 2002 (CERN, Geneva, 2002), p. 683.

[15] CSR-Workshop-2001 held in Berlin, see http://www.desy.de/csr/csr_workshop_2002.

[16] See copies of transparencies by M. Borland and P. Piot presented at CSR-Workshop-2001 at http://www.desy.de/ csr/csr_workshop_2002. 